\title{
Estratégias assistenciais para o controle da tuberculose drogarresistente: revisão integrativa da literatura
}

\author{
Care strategies for controlling drug-resistant tuberculosis: integrative literature review
}

Estrategias asistenciales para el control de la tuberculosis farmacoresistente: revisión integradora de la literatura

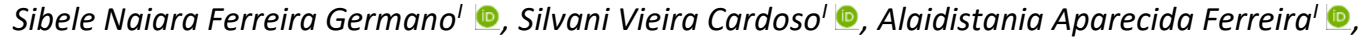 \\ Arinete Véras Fontes Esteves' ㄴ] Marlucia da Silva Garrido' ㄴ?
}

\begin{abstract}
'Universidade Federal do Amazonas, Manaus, AM, Brasil
RESUMO

Objetivo: identificar, na literatura científica, estratégias assistenciais para o controle da tuberculose drogarresistente. Método: revisão integrativa da literatura, com análise de pesquisas relevantes sobre a questão nortedora: Quais são as evidências científicas sobre as estratégias assistenciais para o controle da tuberculose drogarresistente? Busca realizada nas bases Literatura LatinoAmericana e do Caribe em Ciências da Saúde, Medical Literature Analysis, Índice Bibliográfico Espanhol em Ciências da Saúde e Banco de Dados em Enfermagem, entre janeiro e março de 2020. Foram incluídos dez artigos para discussão dos resultados que responderam à questão da pesquisa, atendendo aos critérios de inclusão e exclusão. Resultados: nos estudos publicados nos últimos cinco anos, $80 \%$ abordaram estratégias assistenciais para o controle da tuberculose drogarresistente e $20 \%$ evidenciaram falhas na assistência aos portadores da doença. Conclusão: a revisão da literatura identificou várias estratégias assistenciais para o controle da tuberculose drogarresistente, com destaque para a descentralização do diagnóstico e tratamento compartilhado, possibilitando uma atenção ampliada e integral aos pacientes.

Descritores: Promoção da Saúde; Equipe de Assistência ao Paciente; Assistência Centrada no Paciente; Tuberculose Resistente a Múltiplos Medicamentos.
\end{abstract}

\section{ABSTRACT}

Objective: from the scientific literature, to identify care strategies for controlling drug-resistant tuberculosis. Method: this integrative literature review examined relevant research on the research question - What is the scientific evidence on care strategies for controlling drug-resistant tuberculosis? - by searching Latin American and Caribbean Health Sciences Information, Medical Literature Analysis, Índice Bibliográfico Español en Ciencias de la Salud and Banco de Dados em Enfermagem, between January and March 2020. Ten articles were included in order to discuss findings that answered the research question, after meeting the inclusion and exclusion criteria. Results: of studies published in the past five years, $80 \%$ addressed care strategies for controlling drug-resistant tuberculosis and $20 \%$ revealed shortcomings in care for patients with the disease. Conclusion: the literature review identified several care strategies for controlling drug-resistant tuberculosis, particularly by decentralized diagnosis and shared treatment, allowing expanded, comprehensive patient care.

Descriptors: Health Promotion; Patient Care Team; Patient-Centered Care; Tuberculosis, Multidrug-Resistant.

\section{RESUMEN}

Objetivo: identificar, en la literatura científica, estrategias de asistencia para el control de la tuberculosis farmacorresistente. Método: se trata de una revisión integradora de la literatura, con análisis de investigaciones relevantes sobre la cuestión rectora: ¿Cuáles son las evidencias científicas sobre las estrategias de asistencia para el control de la tuberculosis farmacorresistente? La búsqueda fue realizada en las bases Literatura Latinoamericana y del Caribe en Ciencias de la Salud, Medical Literature Analysis, Índice Bibliográfico Español en Ciencias de la Salud y Banco de Datos en Enfermería, de enero a marzo de 2020. Se incluyeron diez artículos para discutir los resultados que respondieron a la pregunta de la investigación, cumpliendo con los criterios de inclusión y exclusión. Resultados: en los estudios publicados en los últimos cinco años, el $80 \%$ abordó estrategias de atención para el control de la tuberculosis farmacorresistente y el $20 \%$ mostró fallas en la atención de los pacientes con la enfermedad. Conclusión: la revisión de la literatura identificó varias estrategias asistenciales para el control de la tuberculosis farmacorresistente, con énfasis en la descentralización del diagnóstico y tratamiento compartido, permitiendo una atención ampliada e integral a los pacientes.

Descriptores: Promoción de la Salud; Grupo de Atención al Paciente; Atención Dirigida al Paciente; Tuberculosis Resistente a Múltiples Medicamentos.

\section{INTRODUÇÃO}

A tuberculose drogarresistente (TB-DR) é uma forma grave da tuberculose (TB) que pode variar de monorresistência (resistência a um antibiótico de primeira linha) a resistência extensiva (TB-XDR), ou seja, resistência tanto às drogas de primeira linha como resistência adicional a qualquer fluoroquinolona e a pelo menos um dos três

Agradecimentos à Fundação de Amparo à Pesquisa do Amazonas (Fapeam): Programa de Apoio à Pós-graduação Stricto Sensu POSGRAD 2020. Resolução N. 006/2020. Autora correspondente: Sibele Naiara Ferreira Germano. E-mail: sibelenaiaraferreiragermano@gmail.com

Editora Científica: Cristiane Helena Gallasch; Editora Associada: Sonia Acioli de Oliveira 
medicamentos injetáveis de segunda linha (amicacina, canamicina ou capreomicina). Ressalta-se que, mesmo com o esquema terapêutico preconizado e padronizado, a ocorrência da resistência às drogas antituberculose vem aumentando a gravidade da doença ${ }^{1}$.

O aumento galopante da TB-DR nos últimos anos traz preocupações às autoridades e gestores da área da saúde. Estes reconhecem o risco potencial de tornar ineficazes os esquemas terapêuticos disponíveis, impondo a necessidade de utilizar estratégias assistenciais adequadas para o controle da doença que vão além do tratamento medicamentoso ${ }^{2}$.

A TB-DR é complexa e envolve fatores sociais, culturais, políticos e econômicos que favorecem o desenvolvimento da resistência a diferentes fármacos. Além disso, ocorre ainda a própria contaminação com bactérias já resistentes ${ }^{1}$.

Atualmente, 30 países concentram 95\% dos casos mundiais de TB, entre os quais se encontra o Brasil, como representante das Américas ${ }^{3}$. Estima-se que, em 2017, no mundo, 7,1\% dos casos novos de TB e 7,9\% dos casos previamente tratados apresentaram resistência aos principais fármacos disponíveis. $O$ número de casos de TB-DR aumenta a cada ano nos países que concentram os casos de TB, como Brasil, China, Rússia, Índia, entre outros ${ }^{1-3}$.

Para reverter o quadro atual, a Organização Mundial da Saúde (OMS) propôs a Estratégia pelo Fim da Tuberculose no mundo, incluindo o acesso a assistência adequada e a adesão ao tratamento gratuito disponível nos serviços de saúde. Para tanto, deve ser considerado o contexto do paciente com TB, o fortalecimento da atenção humanizada e a integração com a equipe multiprofissional. Nessa estratégia, a enfermagem possui o protagonismo histórico no acolhimento e na adesão do paciente ao tratamento da doença ${ }^{4}$.

É necessário que o enfermeiro, junto com os demais profissionais que trabalham na assistência ao paciente com TB, conheça as estratégias assistenciais de adesão ao tratamento. É indispensável aumentar a integração e valorização do indivíduo acometido pela doença como sujeito corresponsável pelo seu tratamento, visando gerar qualidade assistencial, diminuir a taxa de abandono e, consequentemente, quebrar a cadeia de transmissão da doença, que se configura como relevante fenômeno social ${ }^{5}$.

Nesta perspectiva, é importante conhecer as estratégias atuais e inovadoras da assistência proporcionada aos pacientes com TB-DR, que os tornem protagonistas de seu cuidado na construção de saberes e ações para o alcance da cura $^{4}$. O objetivo deste estudo é identificar, na literatura científica, estratégias assistenciais para o controle da tuberculose drogarresistente.

\section{MÉTODO}

Trata-se de uma revisão integrativa da literatura com abordagem descritiva para compreensão completa do fenômeno, por meio da análise de pesquisas relevantes que dão suporte à tomada de decisão na assistência. 0 desenvolvimento desta pesquisa ocorreu em seis etapas: 1ㅇ) identificação do tema e definição da pergunta nortedora da pesquisa; 2ㅇ) busca na literatura e seleção criteriosa da amostragem; 3의) categorização dos dados; 4의) análise crítica dos estudos incluídos; 5ㅇ) interpretação dos resultados da revisão integrativa; 6ㅇ) relato da revisão e síntese do conhecimento ${ }^{6,7}$.

A questão nortedora da pesquisa surgiu da estratégia PICO, acrônimo no idioma inglês que, em português, significa: $P$ - população, I - intervenção, C - comparação, $O$ - resultados ${ }^{7}$. Neste estudo foi atribuído para $P$ - pacientes com TB-DR; I - assistência ao paciente com TB-DR; C - não foi aplicado, por não se tratar de estudo comparativo; e O controle da TB-DR. Desse modo, a questão norteadora da pesquisa foi assim enunciada: Quais são as evidências científicas sobre as estratégias assistenciais para o controle da tuberculose drogarresistente?

A busca para a seleção das publicações ocorreu nos meses de janeiro a março de 2020 em quatro bases de dados: LILACS (Literatura Latino-Americana e do Caribe em Ciências da Saúde), MEDLINE (Medical Literature Analysis), IBECS (Índice Bibliográfico Espanhol em Ciências da Saúde), e BDENF (Banco de Dados em Enfermagem).

A coleta de dados foi realizada por meio de consultas nas bases de dados acessadas pelo portal da Biblioteca Virtual em Saúde (BVS) com a ajuda de uma bibliotecária, utilizando os descritores em Ciências da Saúde (DeCS): Tuberculose Resistente a Múltiplos Medicamentos e seus respectivos sinônimos: Tuberculose Resistente a Drogas, Tuberculose Farmacorresistente; Assistência Centrada no Paciente; Promoção da Saúde; Equipe de Assistência ao Paciente.

Foram realizadas quatro estratégias de busca nas bases de dados com o uso do descritor de assunto, utilizando os operadores booleanos AND e OR: 1) "Tuberculose Resistente a Múltiplos Medicamentos" OR "Tuberculose Resistente a Drogas" OR "Tuberculose Farmacorresistente" AND "Assistência Centrada no Paciente"; 2) Tuberculose Resistente a Múltiplos Medicamentos OR "Tuberculose Resistente a Drogas" OR “Tuberculose Farmacorresistente" AND "Promoção da Saúde"; 3) "Tuberculose Resistente a Múltiplos Medicamentos" OR "Tuberculose Resistente a Drogas" OR "Tuberculose Farmacorresistente" AND "Equipe de Assistência ao Paciente"; 4) Tuberculose Resistente a Múltiplos Medicamentos OR "Tuberculose Resistente a Drogas" OR “Tuberculose Farmacorresistente" AND "Assistência Centrada no Paciente" OR “Promoção da Saúde”. 
Os critérios de inclusão na revisão de literatura foram assim estabelecidos: artigos originais publicados na íntegra nos últimos cinco anos (2015 a 2019), disponíveis eletronicamente, em português, inglês e/ou espanhol. Foram excluídos estudos duplicados, revisões de literatura, estudos reflexivos, relatos de experiência e os que não possuíssem aderência à questão norteadora da pesquisa.

Os artigos foram selecionados em três etapas realizadas entre pares. Na primeira etapa, que teve início com a busca nas bases de dados selecionadas cruzando os descritores, foram identificados 8.041, dos quais 1.665 preencheram os critérios de inclusão. Na segunda etapa, foram excluídos 1.558 artigos após a leitura do título principal. Aplicando-se os critérios de exclusão, 97 artigos foram excluídos após a leitura dos resumos, por não possuírem aderência à questão nortedora de pesquisa, resultando em 10 artigos para a terceira etapa. Nesta ocorreu a leitura na íntegra conforme demonstrado no fluxograma de seleção dos estudos baseado no modelo PRISMA ${ }^{7}$ (Figura 1).

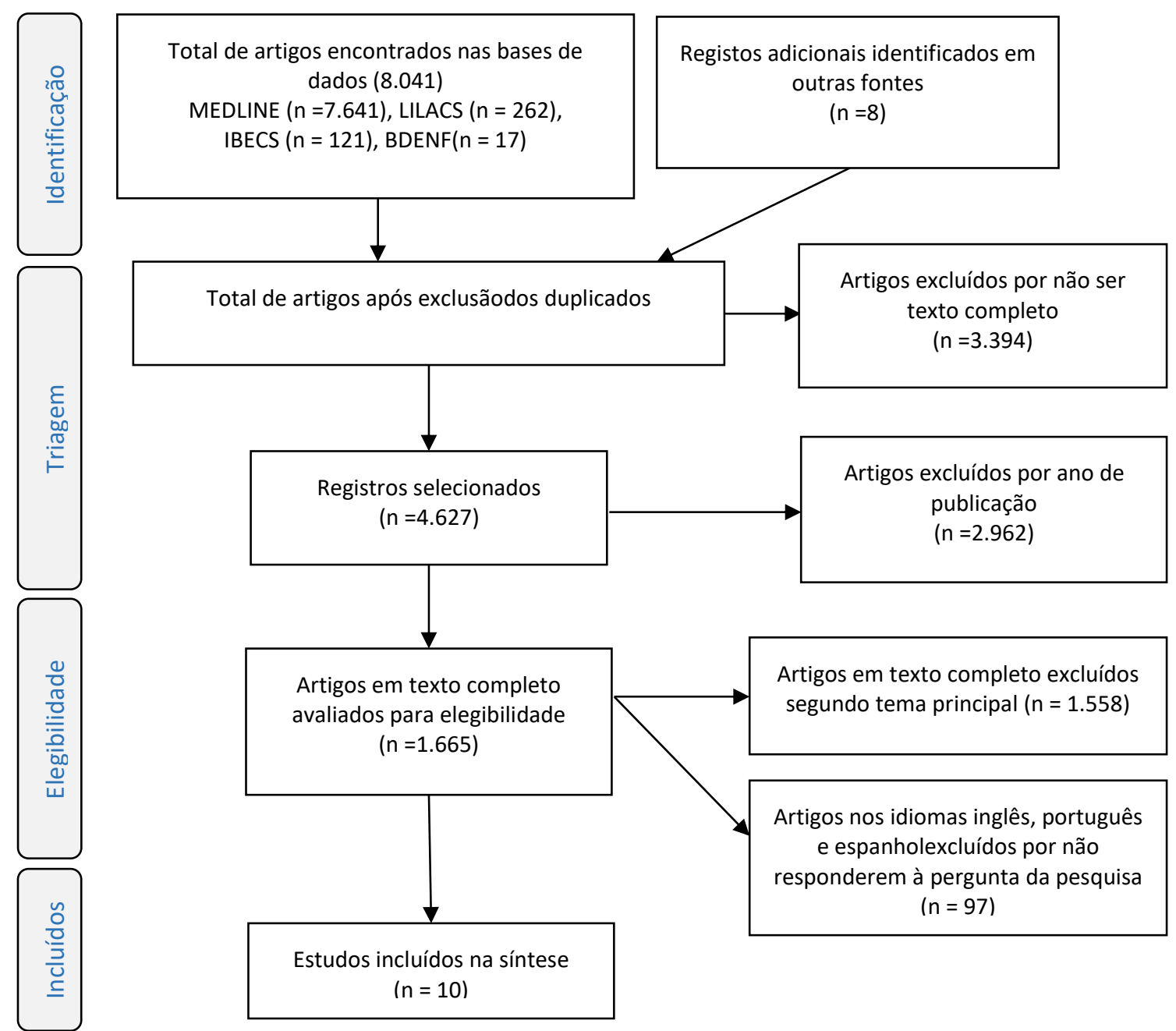

FIGURA 1: Fluxograma de seleção dos estudos baseado no PRISMA7. Manaus, AM, Brasil, 2020

\section{RESULTADOS E DISCUSSÃO}

Os artigos incluídos nesta revisão integrativa foram publicados no período de 2015 a 2019, em periódicos internacionais. Alguns dos países em que foram realizados os estudos são reconhecidos pela OMS como prioritários na aplicação de estratégias assistenciais para o controle da tuberculose (TB) e da tuberculose drogarresistente (TB-DR). Os nove artigos em inglês e um em espanhol estão descritos na Figura 2, relativamente a título, país e natureza do estudo, objetivo geral, revista onde foi publicado e Qualis-CAPES. 


\begin{tabular}{|c|c|c|c|c|c|}
\hline Título do estudo & $\begin{array}{l}\text { País(es) do } \\
\text { estudo }\end{array}$ & $\begin{array}{c}\text { Natureza do } \\
\text { estudo }\end{array}$ & Objetivo geral & Periódico & Qualis \\
\hline $\begin{array}{l}\text { Quality of tuberculosis care in } \\
\text { high burden countries: the } \\
\text { urgent need to address gaps in } \\
\text { the care cascade }\end{array}$ & Índia & $\begin{array}{l}\text { Revisão } \\
\text { Sistemática }\end{array}$ & $\begin{array}{l}\text { Descrever as evidências atuais } \\
\text { sobre a qualidade do tratamento } \\
\text { da TB em países de alta carga. }\end{array}$ & $\begin{array}{l}\text { Int. J. Infect. } \\
\text { Dis. }\end{array}$ & A3 \\
\hline $\begin{array}{l}\text { Tuberculosis care strategies and } \\
\text { their economic consequences } \\
\text { for patients: the missing link to } \\
\text { end tuberculosis } 9\end{array}$ & Etiópia & $\begin{array}{l}\text { Estudo } \\
\text { transversal }\end{array}$ & $\begin{array}{l}\text { Determinar as conseqüências } \\
\text { econômicas da estratégia DOT } \\
\text { para pacientes com TB. }\end{array}$ & $\begin{array}{l}\text { Infect. Dis. } \\
\text { Poverty. }\end{array}$ & A1 \\
\hline $\begin{array}{l}\text { Using Patient Pathway } \\
\text { Analysis to Design Patient- } \\
\text { centered Referral Networks } \\
\text { for Diagnosis and Treatment } \\
\text { of Tuberculosis: The Case of } \\
\text { the Philippines }{ }^{10}\end{array}$ & Filipinas & $\begin{array}{l}\text { Metodologico } \\
\text { de análise do } \\
\text { caminho do } \\
\text { paciente (APP) }\end{array}$ & $\begin{array}{l}\text { Fornecer evidência sobre as } \\
\text { possíveis lacunas na prestação de } \\
\text { serviços que podem estar } \\
\text { contribuindo para a prevalência } \\
\text { persistente de tuberculose. }\end{array}$ & J. Infect. Dis. & A1 \\
\hline $\begin{array}{l}\text { Team approach to manage } \\
\text { difficult-to-treat TB cases: } \\
\text { Experiences in Europe and } \\
\text { beyond }^{11}\end{array}$ & $\begin{array}{l}\text { Portugal, } \\
\text { Bielorrússia, } \\
\text { Bélgica, } \\
\text { França, México } \\
\text { e Reino Unido }\end{array}$ & $\begin{array}{l}\text { Multicêntrico } \\
\text { Análise } \\
\text { Comparativa }\end{array}$ & $\begin{array}{l}\text { Descrever as diferentes } \\
\text { experiências com o TB Consilium } \\
\text { tanto em nível internacional, } \\
\text { quanto em alguns dos países } \\
\text { onde essa experiência opera com } \\
\text { sucesso. }\end{array}$ & Pulmonology & A1 \\
\hline $\begin{array}{l}\text { Model of care and risk factors } \\
\text { for poor outcomes in patients } \\
\text { on multi-drug resistant } \\
\text { tuberculosis treatment at two } \\
\text { facilities in eSwatini (formerly } \\
\text { Swaziland), 2011-2013 }\end{array}$ & Suazilândia & $\begin{array}{l}\text { Estudo de } \\
\text { Coorte } \\
\text { Observacional } \\
\text { Retrospectivo }\end{array}$ & $\begin{array}{l}\text { Relatar os resultados do } \\
\text { tratamento de um coorte de } \\
\text { pacientes com TB-MDR inscritos } \\
\text { entre } 2011 \text { e } 2013 \text {. }\end{array}$ & PLoS One & A1 \\
\hline $\begin{array}{l}\text { "Home is where the patient is": } \\
\text { a qualitative analysis of a } \\
\text { patient-centred model of care } \\
\text { for multi-drug resistant } \\
\text { tuberculosis }{ }^{13}\end{array}$ & Uganda & $\begin{array}{l}\text { Qualitativo } \\
\text { exploratório }\end{array}$ & $\begin{array}{l}\text { Determinar a aceitabilidade e } \\
\text { acessibilidade do tratamento } \\
\text { domiciliar com TB-MDR. }\end{array}$ & $\begin{array}{l}\text { BMC Health } \\
\text { Serv. Res. }\end{array}$ & $\mathrm{A} 2$ \\
\hline $\begin{array}{l}\text { Cost-effectiveness of a } \\
\text { comprehensive programme for } \\
\text { drug-resistant tuberculosis in } \\
\text { China }^{14}\end{array}$ & China & $\begin{array}{l}\text { Estudo de } \\
\text { Coorte }\end{array}$ & $\begin{array}{l}\text { Investigar a relação custo- } \\
\text { benefício de um programa } \\
\text { abrangente de tuberculose } \\
\text { resistente a medicamentos } \\
\text { lançado em quatro locais na } \\
\text { China em } 2011 .\end{array}$ & $\begin{array}{l}\text { Bull.World } \\
\text { Health Organ. }\end{array}$ & A1 \\
\hline $\begin{array}{l}\text { "My Favourite Day Is Sunday": } \\
\text { Community } \\
\text { Perceptions of (Drug-Resistant) } \\
\text { Tuberculosis } \\
\text { and Ambulatory Tuberculosis } \\
\text { Care in KaraSuu District, Osh } \\
\text { Province, Kyrgyzstan } 16\end{array}$ & Quirguistão & $\begin{array}{l}\text { Qualitativo } \\
\text { Validação por } \\
\text { triangulação }\end{array}$ & $\begin{array}{l}\text { Compreender a percepção de TB } \\
\text { e TB-DR, a fim de melhorar a } \\
\text { eficácia e aceitação da } \\
\text { intervenção de Médicos Sem } \\
\text { Fronteiras (MSF). }\end{array}$ & PLoS One & A1 \\
\hline $\begin{array}{l}\text { WHO strategies for the } \\
\text { management of drug-resistant } \\
\text { tuberculosis }^{17}\end{array}$ & México & $\begin{array}{l}\text { Qualitativo } \\
\text { Descritivo } \\
\text { Exploratorio }\end{array}$ & $\begin{array}{l}\text { Expor como aplicar as ações } \\
\text { prioridades da OMS para } \\
\text { controlar a Ttuberculose } \\
\text { Multirresistente TBMR. }\end{array}$ & $\begin{array}{l}\text { Arch. } \\
\text { Bronconeumol } \\
\end{array}$ & B1 \\
\hline $\begin{array}{l}\text { 'Whole person' approach } \\
\text { used in complex TB case }{ }^{18}\end{array}$ & Nova Zelândia & Estudo de caso & $\begin{array}{l}\text { Explorar a importância de uma } \\
\text { equipe multidisciplinar (MDT) ao } \\
\text { lidar com um paciente com } \\
\text { tuberculose resistente a } \\
\text { medicamentos. }\end{array}$ & $\begin{array}{l}\text { Kai Tiaki } \\
\text { Nursing New } \\
\text { Zealan }\end{array}$ & B4 \\
\hline
\end{tabular}

Legenda: Ref. - referência.

FIGURA 2: Quadro descritivo dos artigos inclusos no estudo de revisão de literatura, Manaus-AM, Brasil,2020. 
Quanto à área de publicação, um foi em Enfermagem, um em Serviços de Saúde, dois em Epidemiologia, dois em Ciência e Medicina e quatro em Infectologia. Nos estudos realizados, 80\% abordaram estratégias assistenciais para o controle da TB e TB-DR e $20 \%$ evidenciaram falhas na assistência aos portadores da doença acompanhados pelos serviços de saúde.

Os estudos demonstraram que várias são as estratégias assistenciais que podem ser efetivamente utilizadas para a prevenção e controle da TB-DR. Entretanto, as falhas que ocorrem na assistência têm apresentado como consequência o aumento dos casos de TB-DR ao longo do tempo ${ }^{8-10}$.

O acesso ao diagnóstico e ao tratamento adequado da TB-DR nos serviços de saúde é a estratégia central para uma assistência adequada aos pacientes. A ausência ou demora no diagnóstico pelo teste rápido molecular para tuberculose - Xpert MTB/RIF resulta em condutas terapêuticas inapropriadas, que agravam o quadro do paciente. Esse exame é essencial para detectar não só a presença do material genético de Mycobacterium tuberculosis, mas também o principal gene associado à resistência à rifampicina ${ }^{8}$.

Os estudos destacaram que o diagnóstico da TB sem o acesso ao citado teste traz como consequências tratamento e acompanhamento inicial inadequados, favorecendo a transmissão de cepas resistentes. A demora na melhora do quadro clínico leva ao abandono do tratamento pelo paciente, que precisará iniciar um novo, mais longo e com medicamentos mais tóxicos ${ }^{8,10}$.

Após o estabelecimento do diagnóstico e do tratamento adequado e em tempo oportuno, o Tratamento Diretamente Observado (TDO) torna-se estratégia essencial e muito utilizada na atualidade para prevenção e controle da TB-DR, por evitar a utilização de medicação incorreta e interrupção do tratamento. Entretanto, estudo sugere a aplicação de uma estratégia nova do TDO, baseada na realidade dos pacientes em sua comunidade e aplicada pela equipe da Atenção Primária à Saúde (APS), usando um modelo adequado para a provisão dos cuidados complexos ${ }^{8}$.

Nas Filipinas, foi utilizada como estratégia a implantação de um Programa Nacional de Desenvolvimento de Enfermeiras, com treinamento para aplicação do TDO na comunidade. O resultado foi mais capacidade de atendimento clínico em âmbito local e tratamento com a mais complexa linha de regime terapêutico, incluindo fármacos injetáveis de segunda linha aplicados por enfermeiras da APS. Esta estratégia evitou que o paciente realizasse o longo tratamento em centros especializados distantes de sua família e de sua comunidade ${ }^{10}$.

Um estudo multicêntrico, realizado na Bielorrússia, Bélgica, França, México, Portugal e Reino Unido, apontou a utilização do "Global TB Consilium", estratégia tecnológica global de consultoria clínica eletrônica gratuita oferecida pela Global TB Network (GTN), para todos os serviços e programas que trabalham com casos complexos de TB, como a TB-DR e a TB-XDR. O serviço fornece aos médicos opiniões detalhadas e coordenadas de especialistas sobre esses casos complexos, e os especialistas qualificados respondem no prazo máximo de 48 horas após o envio do caso ${ }^{11}$.

A fim de melhorar os resultados clínicos frente à TB-DR em âmbito local, a estratégia tecnológica do TB Consilium ajuda os vários países no treinamento e aconselhamento dos profissionais que tratam esses pacientes em sua comunidade. Além disso, promovem o acompanhamento do caso de forma unificada, diminuindo os riscos de erros de diagnóstico e acompanhamento inadequado dos portadores ${ }^{11}$. Esse procedimento demonstra que a tecnologia é fundamental para o manejo adequado e uniforme dos casos de TB-DR nos diferentes contextos mundiais.

O diagnóstico, tratamento e acompanhamento realizados por profissionais treinados, com uso de tecnologia no contexto local, aumenta a taxa de cura, diminuindo a baixa adesão ao tratamento da TB-DR que é frequentemente atribuída a modelo de atendimento centralizado em alguns centros especializados. Com isso, os pacientes precisam sair da aréa ou comunidade em que vivem, em busca desses centros que, em sua maioria, estão localizados longe do seu domicílio. Essa dificuldade leva muitos a abandonarem o tratamento por falta de condições financeiras para custear o deslocamento e manterem suas famílias ${ }^{10}$.

Desse modo, é evidente a necessidade de uma assistência descentralizada para o nível local e centrada no paciente e na comunidade para o controle da TB-DR. Estudo realizado na Etiópia recomenda essa estratégia para o tratamento da TB-DR. Demonstra também que muitos pacientes abandonam o tratamento, chegando a desenvolver a Tuberculose Extensivamente Resistente (TB-XDR), por terem que se submeter a tratamento complexo, longe de seu contexto familiar e comunitário, sem dispor de condições sociais e econômicas ${ }^{9}$. Em consequência das dificuldades vivenciadas pelos pacientes, após o diagnóstico de TB-DR, apenas a metade deles realiza o TDO e conclui o tratamento com sucesso ${ }^{8-9}$.

Outro aspecto de maior complexidade, como o manejo de pacientes portadores de TB-DR na população com altas taxas de coinfecção pelo vírus da imunodeficiência humana (HIV), exige regimes de tratamento mais eficazes e mais tolerados. Indica-se o modelo abrangente de cuidados centrados no paciente, proporcionado pelas equipes de saúde no domicílio, incluindo apoio psicossocial. Esse modelo tem contribuído com resultados efetivos no tratamento dessas pessoas ${ }^{12}$. 
A assistência centrada no paciente de TB-DR também foi avaliada em uma comunidade rural em Uganda, com ênfase em seu contexto domiciliar. Nesse estudo, os pacientes da comunidade rural e a equipe multiprofissional elegeram a assistência centrada no paciente no seu domicílio como o local preferido por ambos, em comparação com a assistência hospitalar, por três razões principais: o domicílio é visto como mais propício à recuperação do paciente, permite maior apoio psicossocial e permite mais tempo livre para pacientes e cuidadores realizarem outras atividades, diminuindo o estresse psicológico ${ }^{13}$.

Os estudos evidenciam que todos os países, mesmo aqueles com recursos limitados, devem aplicar a estratégia assistencial do atendimento integral aos pacientes. Isto implica em fornecer ampla cobertura dos serviços de saúde com incentivos financeiro e nutricional, para evitar gastos posteriores em consequência do abandono do tratamento. $O$ fato de o paciente ser afastado de suas atividades laborais devido à doença faz com que ele enfrente graves problemas financeiros e psicológicos ${ }^{9,10,12}$.

Numa investigação abrangente realizada na China, por meio de um programa com 73 pacientes, baseada em uma revisão detalhada dos registros médicos após esses terem completado seis meses de tratamento, destacou-se a efetividade do programa, por incluir triagem rápida, atendimento padronizado e proteção financeira, melhorando os resultados individuais da TB-DR de maneira efetiva ${ }^{14}$

Outro estudo realizado na eSwatini (antiga Suazilândia) com pacientes de TB-DR demonstrou que os cuidados administrados por uma clínica de base comunitária para os portadores dessa doença em seu domicílio mostrou-se eficaz em várias configurações, com altas taxas de cura. Esta estratégia é viável e segura, permitindo que fatores mais amplos de influência na saúde sejam abordados, incluindo o apoio psicossocial. Além disso, modelos de assistência comunitária são de três a quatro vezes mais baratos que as abordagens hospitalares ${ }^{12}$

Estudo realizado no Brasil corrobora os demais estudos, ao destacar que a acessibilidade ao serviço de saúde deve ser valorizada, para a realização do diagnóstico e do tratamento efetivo. Quanto maior o número de unidades básicas de saúde e de profissionais da APS capacitados para aplicar as estratégias de controle da TB, mais eficaz será a busca e a detecção de casos e mais precocemente o tratamento e a supervisão são iniciados, favorecendo a cura e a quebra da cadeia de transmissão ${ }^{15}$.

A busca ativa e a notificação de casos novos de TB é uma estratégia essencial que deve ser promovida pela APS. Os sintomáticos respiratórios, juntamente com os famíliares, devem ser motivados a realizar o exame e, se confirmada a doença, realizar o tratamento o mais rápido possível, com um esquema terapêutico efetivo e com o menor tempo possível de tratamento. Devem ser sempre utilizadas estratégias de conscientização adaptadas a cada grupo vulnerável ${ }^{16}$.

A promoção da saúde também é destacada nos estudos como uma importante estratégia assistencial que deve ser trabalhada durante toda a assistência ao paciente. Estudo enfatiza que a TB, mesmo na forma resistente, é curável desde que diagnósticada e tratada corretamente. São indispensáveis acompanhamento e orientações abrangentes, que expliquem a doença e todo o seu percurso do tratamento até a cura, fornecidas pela equipe multiprofissional, como parte da construção do plano de cuidado ${ }^{13}$

Alguns estudos enfatizaram as estratégias recomendadas pela OMS para controlar a TB-DR, destacando-se quatro delas: prevenção da TB-DR, administrando tratamento de alta qualidade para pacientes com TB sensível; expansão dos testes rápidos para deteç̧ão da TB-DR; oferta do acesso intermediário para um tratamento eficaz e uma atenção adequada; e prevenção da transmissão, usando o controle da infecção nas instituições de saúde 8,10,12,17.

Outra estratégia assistencial muito importante é a abordagem com a equipe muldisciplinar de atendimento aos portadores de TB-DR, para a assistência adequada: realizar reuniões regulares; favorecer a revisão dos cuidados aos doentes; permitir a abordagem integral, centrada no paciente, com comunicação clara em equipe; contribuir para o planejamento dos cuidados e assistência adequada; fortalecer o vínculo entre o profissional e o usuário ${ }^{18}$.

Os estudos revelaram falhas assistenciais, tais como: acesso ao diagnóstico e tratamento tardio, centralização do diagnóstico e tratamento da TB-DR em centros especializados, uso inadequado dos esquemas terapêuticos, ausência de estratégias para o combate das vulnerabilidades sociais, ausência de educação permanente para as equipes de saúde e de educação em saúde para os portadores da doença, famíliares e comunidade ${ }^{8-10}$.

Para as falhas identificadas, as estratégias utilizadas para superação foram: diagnóstico e tratamento compartilhado entre as instituições de referência para TB-DR e a APS; busca ativa de sintomáticos respiratórios de forma permanente com acesso ao teste rápido molecular para tuberculose na APS; TDO baseado na comunidade; educação permanente da equipe multiprofissional para o atendimento clínico e administração dos medicamentos; utilização de tecnologias da informação como o TB Consilium para o manejo dos casos de forma adequada e uniforme; educação em saúde para os usuários, familiares e comunidade, além de suporte financeiro, nutricional e psicológico aos portadores da doença ${ }^{8-14,16-18}$. 
Ressalta-se que, além do tratamento farmacológico, deve ser valorizada a qualidade dos cuidados assistenciais rotineiramente oferecidos aos portadores de TB-DR nos diversos países do mundo, em especial nos países de alta carga de tuberculose, nos setores público e privado, com estratégias que convertam os pacientes em aliados, em todas as etapas de construção do seu plano de cuidado assegurando cuidado e tratamento universal, igualitário e contínuo para o controle da doença ${ }^{12}$.

\section{Limitações do estudo}

Destaca-se como limitação desse estudo, a escassez de estudos sobre a temática nas fontes de dados selecionadas, podendo haver mais estudos em outras fontes de informação não utilizadas nesta pesquisa. Também não se identificou nenhuma tecnologia educacional para os portadores da doença, mesmo sabendo-se da sua importância para o empoderamento e acompanhamento dos usuários na rede de atenção à saúde. Recomenda -se que outras pesquisas sejam realizadas com a ampliação das buscas nas bases de dados, que permita o acesso a um leque maior de conhecimentos que possam fortalecer a utilização de estratégias fundamentais para a prevenção e controle da TB-DR.

\section{CONCLUSÃO}

Por meio desta revisão da literatura foi possível identificar várias estratégias para o controle da TB-DR, com destaque para a descentralização do diagnóstico e realização do tratamento compartilhado, possibilitando uma atenção ampliada e integral aos pacientes. Essas estratégias devem ser aplicadas em todos os serviços de saúde, visando eliminar falhas assistenciais que contribuem para propagar a doença.

O paciente deve ser o centro do plano de cuidado, como protagonista e corresponsável pelo seu tratamento e o bemestar de sua família e comunidade, devendo ser respeitadas as suas peculiaridades e potencialidades socioculturais.

\section{REFERÊNCIAS}

1. Ballestero JGA, Lima MCRAA, Garcia JM, Gonzales RIC, Sicsú AN, Mitano F, et al. Control and management strategies in multidrug-resistant tuberculosis: literature review. Rev. Panam. Salud. [Ineternet]. 2019 [cited 2020 Mar 25]; 43:e20. DOI: https://doi.org/10.26633/RPSP.2019.20.

2. Jacobs MG, Junior VLP. Charecterization of drug-resistant tuberculosis in Brasil, 2014. Epidemiol. Serv. Saúde. [Ineternet]. 2019 [cited 2020 Mar 26]; 28(3):e2018294. DOI: https://doi.org/10.5123/s1679-49742019000300014.

3. World Health Organization. Global tuberculosis report 2019. Geneva: WHO; 2019 [cited 2020 Jun 28]. Available from: https://apps.who.int/iris/bitstream/handle/10665/329368/9789241565714-eng.pdf.

4. Temoteo RCA, Carvalho JBL, Lira ALBC, Lima MA, Sousa YG. Nursing in adherence to treatment of tuberculosis and health Technologies in the contexto of primary care. Esc. Anna Nery. [Ineternet]. 2019 [cited 2020 Mar 22]; 23(3):e20180321. DOI: http://dx.doi.org/10.1590/2177-9465-ean-2018-0321.

5. Sicsú AN, Gonzales RIC, Mitano F, Sousa LO, Silva LMC, Ballestero JGA, et al. Nursing practices centered on individuals with tuberculosis: an interface with democracy. Rev. Bras. Enferm. [Ineternet]. 2019 [cited 2020 Mar 22]; 72(5):1284-90. DOI: http://dx.doi.org/10.1590/0034-7167-2017-0380.

6. Botelho LLR, Cunha CCA, Macedo M. O método da revisão integrativa nos estudos organizacionais. Gestão e Sociedade. [Ineternet]. 2011 [cited 2020 Jan 18]; 5(11): 121-36. DOI: https://doi.org/10.21171/ges.v5i11.1220.

7. Galvão TF, Pansani TSA, Harrad D. Principais itens para relatar revisões sistemáticas e meta-análises: a recomendação PRISMA. Epidemiol. Serv. Saúde. [Ineternet]. 2015 [cited 2019 Jun 14]; 24(2):335-42. DOI: https://doi.org/10.5123/S167949742015000200017.

8. Cazabona D, Alsdurfa H, Satyanarayanaa S, Nathavitharanab R, Subbaramanc R, Daftary A, et al. Quality of tuberculosis care in high burden countries: the urgent need to address gaps in the care cascade. Int. J. Infect. Dis. [Ineternet]. 2017 [cited 2020 Feb 5]; 56:111-6. DOI: http://dx.doi.org/10.1016/j.ijid.2016.10.016.

9. Getahun B, Wubie M, Dejenu G, Manyazewal T. Tuberculosis care strategies and their economic consequences for patients: the missing link to end tuberculosis. Infect. Dis. Poverty. [Ineternet]. 2016 [cited 2020 Feb 5]; 5(1):93. DOI: https://doi.org/10.1186/s40249-016-0187-9.

10. Garfin C, Mantala M, Yadav R, Hanson CL, Osberg M, Hymoff A, et al. Using Patient Pathway Analysis to Design Patient-centered Referral Networks for Diagnosis and Treatment of Tuberculosis: The Case of the Philippines. J. Infect. Dis. [Ineternet]. 2017 [cited 2020 Mar 5]; 216(Suppl 7):S74077. DOI: https://doi.org/10.1093/infdis/jix391.

11. Ambrosioa D, Bothamleyc G, Luna JAC, Duartef R, Guglielmettig L, Torricoi MM, et al. Team approach to manage difficult-totreat TB cases: Experiences in Europe and beyond. Pulmonology. [Ineternet]. 2018 [cited 2020 Feb 15];24(2):132-41. DOI: https://doi.org/10.1016/j.rppnen.2017.10.005.

12. Verdecchia M, Keus K, Blankley S, Vambe D, Ssonko C, Piening T, et al. Model of care and risk factors for poor outcomes in patients on multi-drug resistant tuberculosis treatment at two facilities in eSwatini (formerly Swaziland), 2011-2013. PLoS One. [Ineternet]. 2018 [cited 2020 Mar 12]; 13(19):e0205601. DOI: https://doi.org/10.1371/journal.pone.0205601. 
13. Horter S, Stringer B, Reynolds L, Shoaib M, Kasozi S, Casas EC, et al. "Home is where the patient is": a qualitative analysis of a patient-centred model of care for multi-drug resistant tuberculosis. BMC Health Serv. [Ineternet]. Res. 2015 [cited 2020 Feb 20]; 14:81. DOI: https://doi.org/10.1186/1472-6963-14-81.

14. Fitzpatrick C, Hui Z, Lixia W, Renzhong L, Yunzhou R, Mingting C, et al. Cost-effectiveness of a comprehensive programme for drug-resistant tuberculosis in China. Bull. World Health Organ. [Ineternet]. 2015 [cited 2020 Jan 25]; 93(11):775-84. DOI: http://dx.doi.org/10.2471/BLT.14.146274.

15. Benetti KV, Farias SNP, Souza MHN, Mauro MYC, Medeiros CRS, Parreira PMD. Health service performance in tuberculosis care in the Family Health Strategy. [Ineternet]. Rev. enferm. UERJ. 2018 [cited 2020 Sep 2]; 26:e31643. DOI: http://dx.doi.org/10.12957/reuerj.2018.31643.

16. Burtscher D, Bergh RV, Toktosunov U, Angmo N, Samieva N, Arechaga EPR. "My Favourite Day Is Sunday": Community Perceptions of (Drug-Resistant) Tuberculosis and Ambulatory Tuberculosis Care in Kara Suu District, Osh Province, Kyrgyzstan. PLoS One. [Ineternet]. 2016 [cited 2020 Mar 10]; 11(3):e0152283. DOI: https://doi.org/10.1371/journal.pone.0152283.

17. Rendon A, Centis R, D'Ambrosio L, Migliori GB. WHO strategies for the management of drug-resistant tuberculosis. Arch. Bronconeumol. [Ineternet]. 2017 [cited 2020 Mar 8]; 53(3):95-7. DOI: https://doi.org/10.1016/j.arbres.2016.07.015.

18. Vause A, Aspinall C. 'Whole person' approach used in complex TB case. Kai Tiaki Nurs. N Z. 2015 [cited 2020 Feb 28]. 21(8):30-1. Available from: http://www.rph.org.nz/resources/publications/whole-person-approach-used-in-complex-tb-case-journalarticle.pdf. 